\title{
Optimizing Van der Waals Forces For FIB ex situ Lift Out
}

\author{
Lucille A. Giannuzzi ${ }^{1}$ and Trevor Clark $^{2}$ \\ ${ }^{1 .}$ EXpressLO LLC, Lehigh Acres, FL 33971 USA. \\ ${ }^{2}$ The Pennsylvania State University, University Park, PA 16802 USA.
}

Van der Waals forces are 10-12 orders of magnitude larger than the force of gravity acting on focused ion beam (FIB) prepared specimens and other small particles and fibers, and are the primary adhesion forces responsible for successful, reliable, and rapid ex situ lift out (EXLO) and micromanipulation to suitable specimen carriers [1]. Electrostatic forces which were previously believed to be the operating mechanism for EXLO micromanipulation should be avoided whenever possible [1]. A glass probe is preferred for EXLO since it may be easily drawn to a pointed tip (i.e., $\sim 1 \mu \mathrm{m}$ or less) and remains strong with some flexibility during the manipulation process. Conversely, metal probe tips may easily curl and deform during the manipulation process and therefore are not ideal.

Excessive electrostatic attractive forces between the probe and specimen can make it difficult to release the specimen from the probe [1]. Likewise, excessive electrostatic repulsive forces between the probe and specimen may cause the specimen to jump or fly away from the probe, preventing the possibility of successful manipulation. Due to the disadvantages in using metal probes for EXLO, metallizing the glass probe, via e.g., the deposition of a sputtered metallic layer, can reduce or eliminate adverse electrostatic forces [1]. However, the sputter coating process must be repeated three or more times, rotating the glass probe between sputter coating operations, to fully cover the probe. In addition, some labs may not have a sputter coating in house, and thus, a low cost and easy glass probe metallization solution has been developed.

Many researchers in the semiconductor industry use a permanent (i.e., waterproof) ink and/or marker pen (e.g., "Sharpie" or similar) to write over a region of interest to mark, protect, and fill high aspect ratio features prior to FIB milling [2]. The viscous ink consists of a colorant dispersed in a solvent [3] and acts as a conductive coating thick enough to protect against FIB implantation damage. In this paper, we extend this technique to the metallization of EXLO glass probes. Glass rods were pulled as usual and the tip was dipped manually into a vial of permanent ink. Ink was painted on the remaining length of the glass probe and allowed to dry in air prior to using for manipulation (see Figure 1).

EXLO Pick\&Place ${ }^{\mathrm{TM}}$ manipulation using ink coated glass probes were applied to InGaAs coated InP FIB lift out specimens known to experience attractive electrostatic forces [1]. (N.B. The specimens in reference [1] were erroneously referred to as only InGaAs). The specimens are easily lifted out and manipulated with an ink coated probe as shown in figure 2 . In figure $2 a, b$, manipulation to a carbon coated $\mathrm{Cu}$ TEM grid is shown. In figure $2 \mathrm{c}-\mathrm{e}$, lift out and manipulation to a $\mathrm{Cu} \mathrm{EXpressLO}^{\mathrm{TM}}$ grid is shown. Note that figure 2e shows an ion induced secondary electron (FIB) image of the same specimen and region in figure $2 \mathrm{~d}$ where only Van der Waals forces were operational, i.e., no additional deposition layer or glue was used to hold the specimen to the grid. Thus, permanent ink coated glass probes provide a fast and easy metallization method, reducing electrostatic forces and optimizing Van der Waals forces for EXLO and Pick\&Place ${ }^{\mathrm{TM}}$ micromanipulation processes [4]. 


\section{References:}

[1] L.A. Giannuzzi et al., Microsc. Microanal., 21 (2015) p.1032.

[2] B. Tracy et al., Microsc. Microanal., 15(2) (2009) p.314.

[3] https://en.wikipedia.org/wiki/Permanent_marker

[4] FIB work was performed on a FIB 200 in collaboration with TSS Microscopy.

\section{(a)}

(b)

\section{$1 \mathrm{~mm}$}

Figure 1. Pulled glass tips (a) coated with permanent ink and (b) with no coating (as-pulled).

(a)

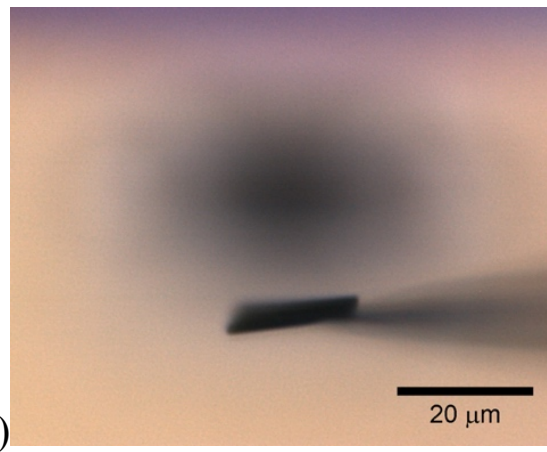

(b)

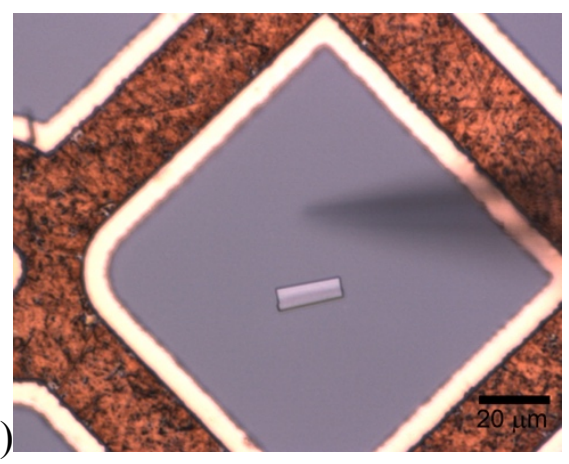

(c)

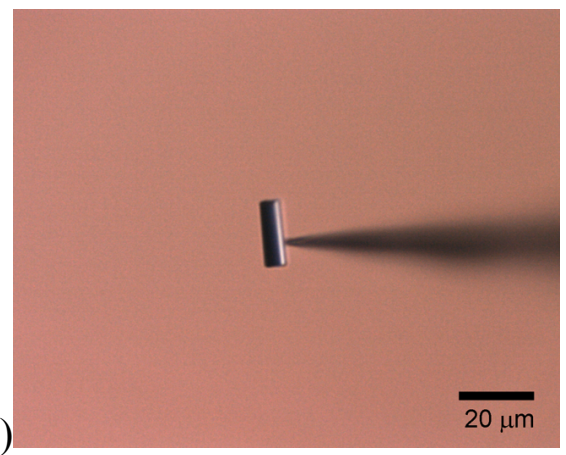

(d) $20 \mu \mathrm{m}$

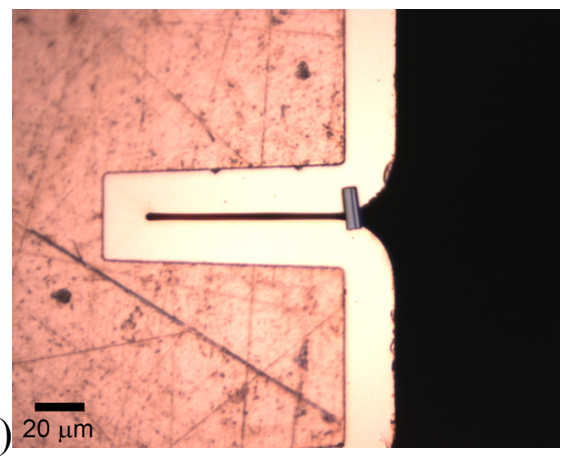

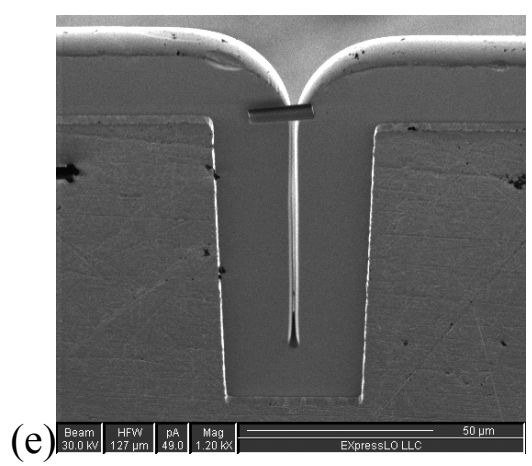

Figure 2. (a-d) Light optical micrographs of the EXLO Pick\&Place ${ }^{\mathrm{TM}}$ manipulation process using ink coated glass tips. $(\mathrm{a}, \mathrm{b})$ Conventional Pick\&Place ${ }^{\mathrm{TM}}$ to a carbon coated $\mathrm{Cu}$ TEM grid. $(\mathrm{c}, \mathrm{d})$ Pick\&Place ${ }^{\mathrm{TM}}$ to a $\mathrm{Cu}$ EXpressLO ${ }^{\mathrm{TM}}$ grid. (e) ion induced secondary electron FIB image of the same specimen. 